\title{
La terminología ATIC: consideraciones de uso en la prestación de cuidados
}

\author{
Mª Eulàlia Juvé Udina \\ Doctora en Ciencias Enfermeras. Instituto de Investigación Biomédica de Bellvitge (IDIBELL). Barcelona \\ Fecha de recepción: 6 de septiembre de 2017. Aceptada su publicación: 6 de noviembre de 2017.
}

Resumen

La terminología ATIC se basa en el lenguaje natural de las enfermeras asistenciales, cuyos conceptos se someten a un refinamiento teórico. Tiene una estructura matricial, no taxonómica, y persigue la representación del proceso de prestación de cuidados de forma circular e iterativa, en la que el primer y el último paso es la interacción de la enfermera con la/s persona/s beneficiaria/s de los cuidados. El objetivo de este artículo es plantear las consideraciones y revisar las evidencias del uso de la terminología ATIC en la práctica asistencial. Para ello se ha realizado una adaptación del método mixto, narrativo, contiguo, en el que los resultados de la revisión de los estudios se presentan secuencialmente junto a sus consideraciones esenciales para que el lector elabore sus propias conclusiones. Partiendo de una concepción circular e iterativa del proceso de prestación de cuidados, el eje de valoración de ATIC se ha empleado para construir modelos de datos y se utiliza en la práctica para representar la valoración integral inicial, continuada y focalizada del estado de la persona. Varios estudios avalan su uso.

El eje diagnóstico de ATIC parte de una concepción pragmática del significado del diagnóstico enfermero y propone una red de nuevos conceptos para su uso en la práctica. Los estudios demuestran su usabilidad, precisión y utilidad para la representación del juicio clínico enfermero. El eje intervención de la terminología se nutre de la conceptualización de la práctica asistencial enfermera y de la representación pragmática de sus prestaciones. Varios estudios analizan su uso, el nivel de comprensión de las enfermeras sobre su contenido y la coherencia entre el registro de los diagnósticos, las intervenciones y los resultados. En síntesis, las evidencias que sustentan y demuestran el empleo de ATIC en la práctica asistencial parecen apoyar el escenario de que esta facilita el registro y la visibilización de la aportación de los cuidados enfermeros a los resultados de salud, aunque el método empleado deja las conclusiones a consideración de cada lector.

\section{Palabras clave}

Estandarización de la terminología enfermera; diagnóstico enfermero; valoración enfermera; administración de servicios de salud; historia clínica electrónica; documentación clínica.

\section{Abstrac: \\ ATIC Terminology: Considerations of use in patient care}

ATIC Terminology is based on the natural language of nurses; its concepts are object to theoretical refinement. It has a matrix structure, non-taxonomic, and it aims at representing the patient care process in a circular and recurrent manner, where the first and the last step is the interaction of the nurse with the person receiving care. The objective of this article is to present the considerations and review the evidence on the use of ATIC terminology in patient care. For this aim, there has been an adaptation of the mixed, narrative, continuous method, where the results of the review of studies are presented sequentially with their essential considerations, so that readers can draw their own conclusions. Based on a circular and recurrent concept of the patient care process, the evaluation axis of ATIC has been used to build data models, and it is used in practice to represent the initial, continuous and focused comprehensive evaluation of the person's status. Various studies support its use.

The diagnostic axis of ATIC is based on a pragmatic concept of the meaning of nursing diagnosis, and puts forward a network of new concepts to be used in practice. Studies have demonstrated its usability, accuracy and utility for the representation of nursing clinical judgment. The terminology intervention basis feeds off the conceptualization of healthcare practice by nurses and the pragmatic representation of their patient care. Various studies have analyzed its use, the level of understanding by nurses about its contents, and the consistency between the record of diagnoses, interventions and outcomes. Summing up, the evidence that supports and demonstrates the use of ATIC in patient care practice seems to endorse the scenario that ATIC facilitates the record and visibility of the contribution of patient care by nurses to health outcomes, even though the method used leaves conclusions to be drawn by each reader.

\section{Key words}

Standardization of nursing terminology; nursing diagnosis; nursing assessment; administration of healthcare services; electronic clinical record; clinical documentation.

\section{Introducción}

El autor del prólogo de La Enfermera moderna cita: "(...), y me atrevo a pronosticar que, a medida que el nivel científico e intelectual de este nuevo personal que ahora se crea, vaya ascendiendo, (...) podrán ir desarrollando y amplifican- do muchos de los puntos que en la presente, y con el máximo acierto, no han hecho más que esbozar" (1).

Más de 70 años después, el informe El futuro de la Enfermería concluía que las enfermeras han de poder ejercer su

\section{Dirección de contacto:}

Mª Eulàlia Juvé Udina. Universitat de Barcelona. Campus de Bellvitge. Departamento de Enfermería fundamental y médico-quirúrgica. C/ Feixa Llarga, s/n. 08907 Hospitalet de Llobregat (Barcelona).E-mail: Ialajuve.on@gmail.com

Cómo citar este artículo:

Juvé Udin ME. La terminología ATIC: consideraciones de uso en la prestación de cuidados. Metas Enferm dic 2017/ene 2018; 20(10): 67-76. 
práctica en toda la extensión de su capacidad formativa y ámbito de responsabilidad, obtener los niveles académicos más altos, participar del rediseño del sistema sanitario y mejorar los datos y la información sobre la prestación de cuidados (2).

Para ello, quizás ha llegado el tiempo de entender que, con frecuencia, lo mejor es Ilamar a las cosas por su nombre. En su momento, el uso de eufemismos para denominar muchos de los fenómenos de interés y de responsabilidad de la Enfermería fue probablemente una buena estrategia para avanzar en la denominación de los problemas de salud (PS) de las personas beneficiarias de los cuidados enfermeros. Por ejemplo, el diagnóstico Deterioro del intercambio de gases (3) es consistente con el concepto de hipoxemia: "La hipoxemia, o disminución de la presión parcial de oxígeno en sangre arterial por debajo de $80 \mathrm{mmHg}$, refleja un deterioro del intercambio gaseoso" (4). De hecho, no sería literalmente un eufemismo; se trata de un constructo con un nivel de abstracción más elevado que el del concepto de hipoxemia (4).

Incluso la definición del "diagnóstico enfermero" como "un juicio clínico sobre la respuesta de un individuo a un problema de salud (....)"(3), responde claramente a la necesidad histórica de la Enfermería de no emplear la palabra "diagnóstico" como un disfemismo. "Respuesta" significa reacción o consecuencia. Ergo, toda complicación de una enfermedad debería considerarse una respuesta y bajo este prisma, podría ser considerada, un diagnóstico enfermero (5).

Se sabe que las enfermeras emiten juicios clínicos sobre problemas de salud reales o potenciales, no solo acerca de las respuestas a problemas de salud (PS) (5-7). De hecho, un mismo concepto diagnóstico puede ser un problema de salud en un caso y una respuesta a un PS en otros (Cuadro 1).

Hay problemas de salud que quedan delimitados al ámbito de la responsabilidad médica. Otros se sitúan en el de la responsabilidad enfermera y otros quedan dentro del ámbito de la corresponsabilidad. Se duda que ninguna enfermera pretendiera emitir un juicio clínico para diagnosticar una diabetes o un sarcoma. En cambio, a diario las enfermeras diagnostican y tratan a personas que sufren una hipoglucemia, que presentan una infección en una herida crónica, o que tienen riesgo de experimentar un síndrome postdosis después de la administración de una vacuna o de un anti- cuerpo monoclonal. Lo hacen con conocimiento, evidencia y responsabilidad, sabiendo en qué punto la persona tiene que ser reevaluada por la misma enfermera, por otra enfermera con más pericia, por uno o más médicos, o por todo un equipo multidisciplinar.

De todo ello, de lo que se lleva a cabo (intervenciones), del porqué lo hacemos (diagnósticos) y para qué lo hacemos (resultados) trata la terminología ATIC $(4,8,9)$, un lenguaje enfermero de interfase, orientado a conceptos y desarrollado desde 1992 (4,8). Fundamentado en la conceptualización interpretativa de los elementos metaparadigmáticos disciplinares (10-12), complementario a las clasificaciones tradicionales $(4,8)$, sometido a un proceso formal de validación (13-16), que se sustenta sobre el análisis de la producción científica $(4,14)$, implantado en la práctica asistencial desde 2007 (17,18), reconocido por distintas organizaciones profesionales (9), incorporado en los planes de estudios de varias facultades de Enfermería (19), empleado por otras disciplinas como ejemplo de terminología de interfase (20) y evaluado en términos de uso (16,21), satisfacción (8), contribución a la seguridad clínica (22-23), la comunicación interprofesional (23) y los resultados (22-24). Es una terminología que se basa en el lenguaje natural de las enfermeras asistenciales, cuyos conceptos se someten a un refinamiento teórico (4,25). Dicho lenguaje pretende facilitar y optimizar el registro y la explotación de los datos de la prestación de cuidados, contribuyendo a hacer más visible el impacto estas prestaciones en la salud y la autonomía de los ciudadanos $(4,26)$.

El objetivo de este artículo es revisar algunas consideraciones y evidencias del uso de ATIC en la práctica asistencial, empleando una adaptación del método mixto, narrativo, contiguo, en el que los resultados de la revisión de los estudios se presentan secuencialmente junto a sus consideraciones esenciales para que el lector elabore sus propias conclusiones.

\section{Uso de ATIC en la práctica asistencial: consideraciones y evidencias}

La terminología ATIC tiene una estructura matricial, no taxonómica, y persigue la representación del proceso de prestación de cuidados de forma circular e iterativa, en la que el primer y el último paso es la interacción de la enfermera con la/s persona/s beneficiaria/s de los cuidados.

\section{Cuadro 1. Casos prácticos que ilustran los conceptos de problema de salud y respuesta a un problema de salud}

\section{Viñeta 1. Incontinencia urinaria de esfuerzo}

Una mujer adulta, sana, madre de dos hijas adolescentes, con hábitos de vida saludables, que practica Pilates cuatro días a la semana y refiere pérdidas de orina durante los ejercicios abdominales. Si se profundizara más en la valoración, descartadas otras posibles causas, probablemente se observaría que desconoce las técnicas de contracción-relajación de la musculatura del suelo pélvico durante el ejercicio físico abdominal.

- En este caso, la incontinencia de esfuerzo es un problema de salud; no es una respuesta a ningún problema de salud.

\section{Viñeta 2. Incontinencia urinaria de esfuerzo}

En el segundo caso, una mujer adulta postoperada por un carcinoma de endometrio que sufre pérdidas de orina al toser o estornudar. En este supuesto caso, la debilidad de la musculatura abdominal y de suelo pélvico se asocia al proceso de la enfermedad, al procedimiento quirúrgico y, probablemente, a la propia fatiga y sarcopenia consecuencia del proceso neoplásico.

- En este caso, la incontinencia de esfuerzo es una consecuencia y requerirá un abordaje sintomático o puede precisar un abordaje multidisciplinar para su resolución o estabilización. 


\section{Valoración}

Los elementos del Eje valoración de ATIC se han empleado para construir modelos de datos de valoración (MDV) en distintos sistemas de información asistenciales (SIA), con un $82 \%$ de los elementos empleados en los registros enfermeros de forma habitual $(27,28)$.

Es recomendable que los modelos de datos de valoración inicial, continuada y focalizada construidos con ATIC se adecuen a los principios de no-linealidad, para representar de forma más adecuada los procesos mentales que las enfermeras realizan al evaluar a una persona y su contexto. Las enfermeras no trabajan por tareas, sino cuidando a las personas que atienden. Por tanto, cualquier interacción paciente-enfermera es una oportunidad para valorar, evaluar, o reevaluar su situación y que, durante esa interacción, se reciben, procesan y analizan una gran cantidad de inputs que la enfermera captura, interpreta, descarta, contrasta mentalmente con datos anteriores, prioriza y sintetiza. El registro de los datos de valoración, por completo que pueda ser, siempre será un resumen de lo que esa enfermera ha sido capaz de procesar mentalmente (8).

En uno de modelos de datos de valoración creado a partir de ATIC se destaca que, dado que el estado del paciente no es una foto fija, el lenguaje que se use ha de ser un elemento facilitador de la representación de los MDV, de la creación de sistemas inteligentes en los sistemas de información asistenciales $y$, sobre todo, del registro y el retorno de información a las enfermeras (28).

En un análisis multicéntrico de los registros de episodios de pacientes hospitalizados en ocho centros que emplean esta terminología (Tabla 1), se identificó que las enfermeras llevan a cabo una valoración integral de la persona, puesto que todas las categorías de datos contienen información cumplimentada, aunque los elementos de valoración documentados con mayor frecuencia se corresponden con los de los componentes cardiocirculatorio, homeostático-excretor y actividades de la vida diaria (27).

La fundamentación teórica de ATIC mantiene un posicionamiento ecléctico (10-12). Ello permite a los usuarios de esta terminología emplear los elementos estructurantes de ATIC para agrupar y organizar su modelo de datos de valoración, optar por utilizar elementos agrupadores de un modelo conceptual (Gráfico 1), un esquema propuesto por una teorizadora para agrupar los datos, o incluso combinar varias opciones (Tabla 2).

Tabla 1. ATIC eje valoración. Porcentaje de episodios de pacientes con parámetros de valoración correctamente informados, agrupados por elementos estructurantes ( $n=72.593$; periodo: primer semestre 2016)

Categorías ATIC

Oxigenación y perfusión

Nutrición e hidratación

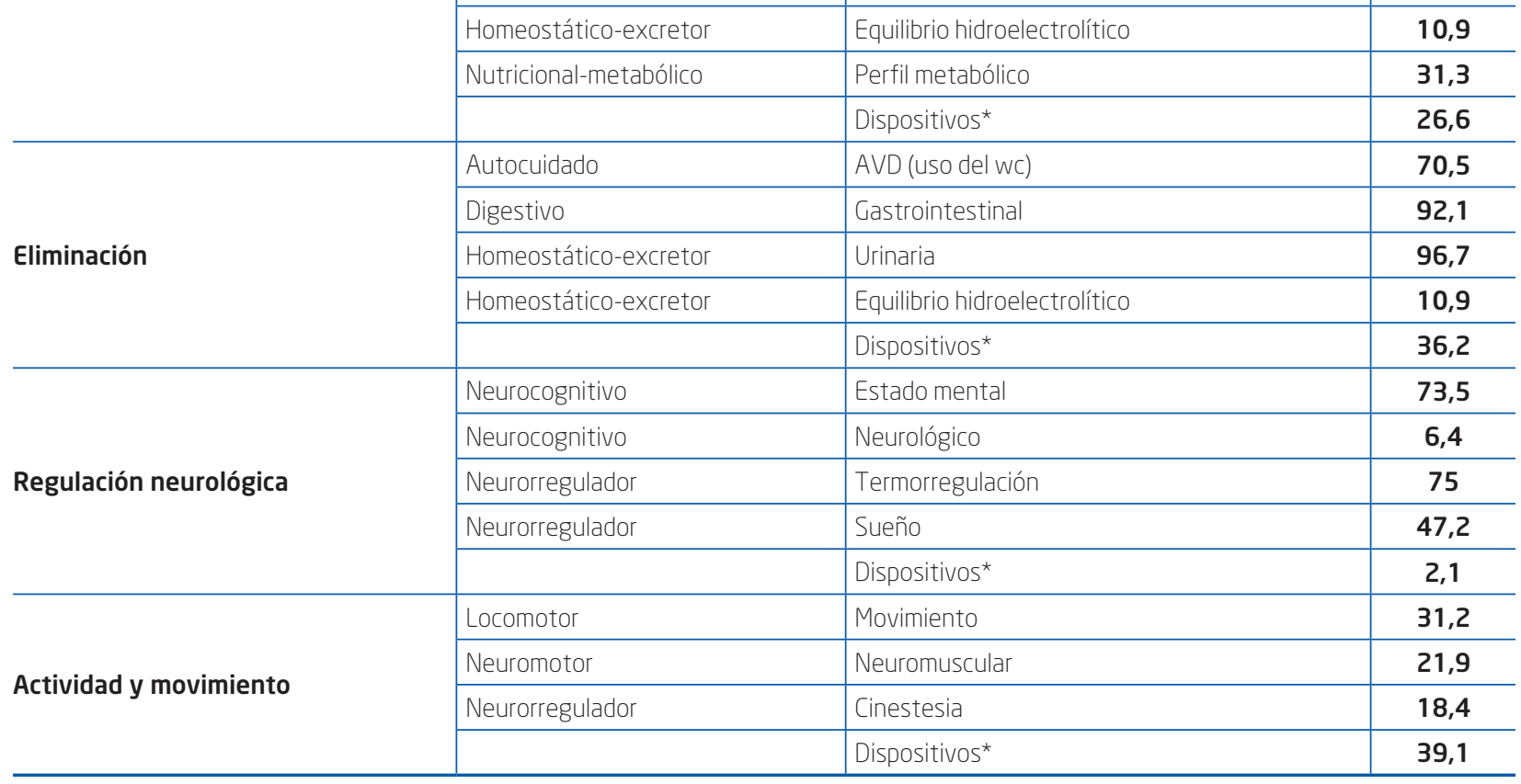


Tabla 1. ATIC eje valoración. Porcentaje de episodios de pacientes con parámetros de valoración correctamente informados, agrupados por elementos estructurantes ( $n=72.593$; periodo: primer semestre 2016) (cont.)

\begin{tabular}{|l|l|l|l|}
\hline Categorías ATIC & Componentes ATIC & Metaconceptos & $\%$
\end{tabular}

Actividad y movimiento

\begin{tabular}{|c|c|c|}
\hline Componentes AIIC & Metaconceptos & $\%$ \\
\hline Autocuidado & Actividad & 50,5 \\
\hline Autocuidado & AVD instrumentales & NV \\
\hline Multicomponente & Energía & 32,4 \\
\hline Neurocognitivo & Comunicación verbal & 50,9 \\
\hline Cultural & Idioma & 49,5 \\
\hline \multirow[t]{2}{*}{ Sensoperceptual } & Sentidos & 8,7 \\
\hline & Dispositivos* & 22,8 \\
\hline Neurocomportamental & Percepción y pensamiento & 29,8 \\
\hline Neurocomportamental & Conducta o comportamiento & 43,5 \\
\hline Autoconceptual & Autoestima & 9,5 \\
\hline Autoconceptual & Imagen corporal & 10,1 \\
\hline Autodeterminación & Conductas de riesgo & 30 \\
\hline Autoconceptual e Interaccional & Identidad y roles & 2,1 \\
\hline Multicomponente & Autopercepción de la salud y estado general & 40,1 \\
\hline Autocuidado & AVD (higiene) & 88,7 \\
\hline Autocuidado & AVD (vestirse) & 45,3 \\
\hline Tegumentario & Cutáneo & 84,9 \\
\hline Tegumentario & Tegumentos & 12,5 \\
\hline Tegumentario & Lesiones & 6,3 \\
\hline \multirow[t]{2}{*}{ Tegumentario } & Heridas & 54,5 \\
\hline & Dispositivos* & 48,7 \\
\hline Reproductor & Regulación hormonal & 5,3 \\
\hline Reproductor & Funcionamiento reproductor & NV \\
\hline Autoconceptual & Sexualidad & 2,9 \\
\hline Adaptativo-emocional & Estado de ánimo & 52,6 \\
\hline Adaptativo-emocional & Afrontamiento y estrés & 44,9 \\
\hline Espiritual & Armonía interior & 13,6 \\
\hline Neurocognitivo & Conocimientos & 40,1 \\
\hline Desarrollo e interaccional & Aprendizaje & 34,2 \\
\hline Adaptativo-emocional & Adherencia & 43,8 \\
\hline Interaccional & Recreación & 3,1 \\
\hline Desarrollo & Tareas de desarrollo y maduración & 9,8 \\
\hline Interaccional & Cuidador & 46,1 \\
\hline Interaccional & Familia & 40,3 \\
\hline Interaccional & Relaciones & 36,3 \\
\hline Doméstico & Hogar & 3,5 \\
\hline Social & Condiciones sociales & 1,6 \\
\hline Social & Determinantes sociales & NV \\
\hline Cultural & Raíces, redes y afiliaciones & NV \\
\hline Cultural & Valores & NV \\
\hline Cultural & Creencias sobre la salud & $<0,5$ \\
\hline Cultural & Preferencias & NV \\
\hline Profesional/laboral & Condiciones laborales & NV \\
\hline Medioambiental & Condiciones ambientales & NV \\
\hline Multicomponente & Alergias e intolerancias & 97,2 \\
\hline
\end{tabular}

Conducta y percepción de la realidad

Higiene y protección tegumentaria

Reproducción y sexualidad

\section{Adaptación}

Relaciones y entorno 


\begin{tabular}{|c|c|c|c|}
\hline $\begin{array}{l}\text { Categorías ATIC Eje Valoración } \\
\text { (Juvé) }\end{array}$ & $\begin{array}{l}\text { Marco Fundamentals of Care } \\
\text { (Kitson) }\end{array}$ & $\begin{array}{l}\text { Necessidades básicas } \\
\text { (Henderson) }\end{array}$ & $\begin{array}{l}\text { Patrones funcionales de salud } \\
\text { (Gordon) }\end{array}$ \\
\hline Oxigenación y perfusión & Respirar & Respirar normalmente & Actividad-ejercicio \\
\hline Nutrición e hidratación & Comer y beber & Comer y beber adecuadamente & Nutricional-metabólico \\
\hline Eliminación & Eliminación & $\begin{array}{l}\text { Eliminar adecuadamente } \\
\text { desechos y secreciones }\end{array}$ & Eliminación \\
\hline \multirow{2}{*}{ Regulación neurológica } & Termorregulación & $\begin{array}{l}\text { Mantener la temperatura dentro } \\
\text { de los límites normales }\end{array}$ & Cognitivo-perceptual \\
\hline & Reposar y dormir & Dormir y descansar & Sueño-descanso \\
\hline Actividad y movimiento & Movimiento & $\begin{array}{l}\text { Moverse y mantener una buena } \\
\text { postura }\end{array}$ & Actividad-ejercicio \\
\hline Comunicación & Comunicación y educación & Comunicarse con los semejantes & Cognitivo-perceptual \\
\hline Conducta y percepción & Seguridad. Toma de decisiones & $\begin{array}{l}\text { Detectar y evitar peligros y evitar } \\
\text { perjudicar a otros* }\end{array}$ & $\begin{array}{l}\text { Cognitivo-perceptual. Adaptación- } \\
\text { autoconcepto. Percepción y } \\
\text { manejo de la salud }\end{array}$ \\
\hline Higiene y protección tegumentaria & Higiene personal y vestido & $\begin{array}{l}\text { Mantener la higiene personal } \\
\text { y proteger los tegumentos. } \\
\text { Vestirse y desvestirse }\end{array}$ & Nutricional-metabólico \\
\hline Sexualidad y reproducción & Expresión de la sexualidad & Comunicarse con los semejantes* & Sexualidad-reproducción \\
\hline Adaptación & Seguridad y confort & $\begin{array}{l}\text { Detectar y evitar peligros y evitar } \\
\text { perjudicar a otros }\end{array}$ & Adaptación-tolerancia al estrés \\
\hline Aprendizaje y desarrollo & $\begin{array}{l}\text { Comunicación y educación* } \\
\text { Ciclo vital }\end{array}$ & $\begin{array}{l}\text { Aprender, descubrir y satisfacer } \\
\text { la curiosidad que conduce a un } \\
\text { desarrollo normal y saludable }\end{array}$ & $\begin{array}{l}\text { Rol-relaciones* Percepción y } \\
\text { manejo de la salud* }\end{array}$ \\
\hline \multirow{4}{*}{ Relaciones y entorno } & Comunicación y educación* & Comunicarse con los semejantes* & Rol-relaciones* \\
\hline & & $\begin{array}{l}\text { Jugar y participar en actividades } \\
\text { recreativas }\end{array}$ & \\
\hline & & Ocuparse para sentirse realizado & \\
\hline & $\begin{array}{l}\text { Dignidad e intimidad. Toma de } \\
\text { decisiones }\end{array}$ & $\begin{array}{l}\text { Reaccionar según las propias } \\
\text { creencias y valores }\end{array}$ & Valores-creencias \\
\hline Multicategoría & Prevención y medicación & & \\
\hline
\end{tabular}

Nota 1. Los sombreados en la tabla no indican la existencia de una correlación lineal directa. Solo orientan similitudes. Los elementos marcados con un * indican repetición.

Nota 2. Además de las categorías, ATIC tiene múltiples elementos estructurantes que se emplean para representar y organizar la valoración. En los centros que emplean ATIC usan los componentes y los metaconceptos del eje valoración como meso y microagrupadores y las necesidades básicas como macro-agrupadores.

Nota 3. Para ampliar información sobre los autores que aparecen en la Tabla 2 consultar: Juvé (ampliar información revisando citas 10-12 del apartado bibliografía). Kitson AL, Muntlin Athlin A, Conroy T. Anything but basic. Nursing's challenges in meeting patients' fundamental care needs. J Nurs Scholarship.2014; 46(5):331 -9. Henderson V. Principios básicos de los cuidados de enfermería. Suiza: Consejo Internacional de Enfermeras; 1971. Gordon M. Practice-based data set for nursing information systems. J Med Systems. 1985; 9:43-55.

Gráfico 1. Porcentaje de elementos de valoración ATIC registrados en la HCE de personas ingresadas en ocho hospitales (2016), agrupados según el Modelo de Necesidades Humanas de Virginia Henderson

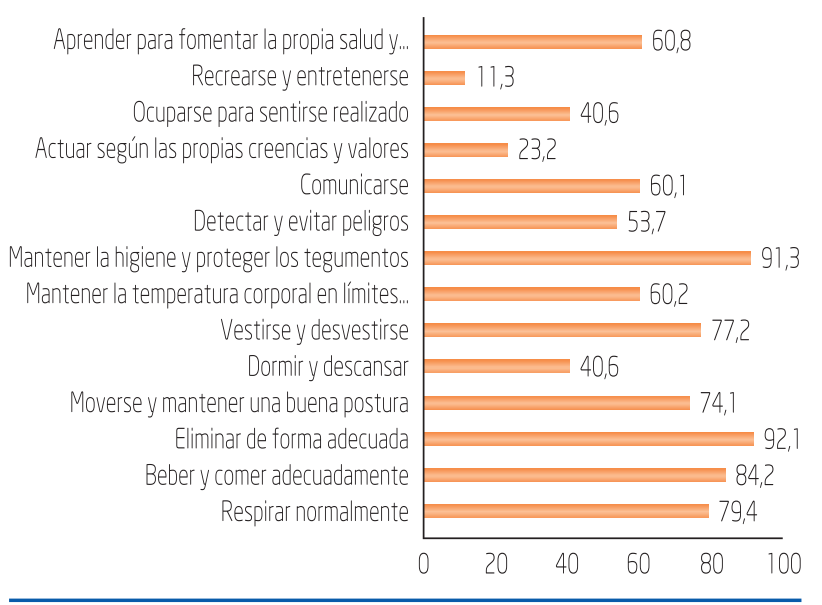

En un estudio analítico, los elementos de la valoración inicial y continuada de ATIC han sido validados como herramienta para la identificación del delirio y del riesgo de delirio en pacientes hospitalizados. Este análisis contribuye a reforzar la utilidad de ATIC no solo para el registro, sino para la mejora de la prestación de cuidados (22,29). Otros estudios evalúan el contenido de los registros de valoración enfermera con ATIC para determinar la presencia de factores individuales de complejidad de cuidados $(30,31)$ y de su impacto en la prevención de los reingresos (32).

\section{Diagnóstico}

Los diagnósticos incluidos contemplan la premisa de que, dentro del ámbito de su responsabilidad, las enfermeras no solo diagnostican respuestas a problemas de salud reales o potenciales en personas, grupos o comunidades, sino que también diagnostican PS. Esta premisa se sustenta sobre la fundamentación teórica $(11,12)$ y el análisis de la produc- 
ción científica enfermera de cada uno de los diagnósticos en ATIC (4).

En la literatura científica son escasos los estudios acerca de la priorización de los elementos de valoración que conducen a un juicio clínico afinado (33). ATIC propone una red de conceptos que ilustran distintas tipologías de diagnósticos como método de apoyo a la priorización, para conseguir reflejar de manera más coherente el juicio clínico diagnóstico o de resultado (Cuadro 2) $(9,16,17)$.

El registro de un diagnóstico es reflejo de la conclusión enfermera sobre el estado, el problema o el resultado de salud del paciente. Esta conclusión está condicionada por el grado de pericia clínica de cada enfermera $(5,8,16)$. En este sentido, la identificación de los resultados de salud es producto del juicio clínico sobre la evolución del estado de salud de la persona (5,8). Por ello, ATIC no contiene un eje de resultados, sino que estos se integran en el eje valoración y en el eje diagnóstico.

En cuanto a su representación práctica en los sistemas de información asistenciales, las enfermeras que utilizan ATIC para reflejar su juicio clínico en los registros, emplean el descriptor (denominación) del diagnóstico, no el formato PES (problema, etiología, signos y síntomas).

\section{Cuadro 2. Red conceptual sobre el diagnóstico enfermero en ATIC}

\section{Diagnóstico enfermero}

Juicio clínico (o la conclusión de varios juicios) sobre el estado de salud del individuo (familia, grupo o comunidad) y las consecuencias y reacciones reales o de riesgo en las distintas dimensiones del individuo y su integralidad, en el contexto de su entorno y su experiencia particular y en el ámbito de la responsabilidad profesional enfermera que incluye también la corresponsabilidad con el beneficiario de los cuidados, sus cuidadores y/o con otros profesionales de la salud $(4,5,8,16)$.

(Todos los conceptos relacionados a continuación son inclusivos de los principios que explicita esta definición).

\section{Diagnóstico principal}

Juicio clínico (o la conclusión de varios juicios) sobre el problema o situación de la persona (familia, grupo o comunidad) que, por su afectación dimensional, gravedad, severidad y/o potencial para producir complicaciones o actuar como factor etiológico, de alto riesgo o desencadenante de otros problemas, genera la mayor necesidad de cuidados enfermeros en términos de inmediatez de su abordaje, intensidad y/o complejidad de cuidados. La priorización del diagnóstico principal no resta importancia a la identificación y al abordaje de los diagnósticos secundarios que puedan existir. Las intervenciones orientadas al abordaje del diagnóstico principal pueden también ser de utilidad en la prestación de cuidados de alguno de los diagnósticos secundarios, pero no deberían repetirse en el registro del plan de cuidados. El diagnóstico enfermero principal puede emplearse como indicador de severidad y/o riesgo de mortalidad $(9,16,17)$.

\section{Diagnóstico secundario}

Juicio clínico (o la conclusión de varios juicios) sobre el problema o situación de la persona (familia, grupo o comunidad) que coexisten con el diagnóstico principal, desde su detección o posteriormente, que generan necesidad de cuidado adicional $(9,16,17)$.

\section{Diagnóstico de agrupación}

Juicio clínico (o la conclusión de varios juicios) sobre el estado de la persona (familia, grupo o comunidad) que agrega un conjunto de problemas que comparten una etiología común. Suele considerarse el diagnóstico enfermero principal $(4,25)$.

Inmadurez fisiológica del neonato

Riesgo de toxicidad multiorgánica

\section{Diagnóstico orientado a la vigilancia}

Juicio clínico (o la conclusión de varios juicios) sobre el curso de un individuo (familia, grupo o comunidad) con riesgo de evolucionar hacia un empeoramiento o agravamiento de su estado de salud, de menor a mayor gravedad, hasta situaciones que ponen en riesgo inminente su integridad. (Véase la conceptualización de los estados de salud) (12).

Este tipo de diagnóstico se centra en el cribado, la vigilancia y la prevención del estado de salud y su deterioro, incluyendo la progresión a estados de agravamiento o exacerbación, y la producción o la recurrencia de complicaciones y eventos adversos.

Debería considerarse el diagnóstico enfermero principal, aunque existan otros diagnósticos que puedan ser significativos, y orientar la prestación de intervenciones que aseguren la valoración continuada del estado de la persona y de intervenciones preventivas que permitan evitar a tiempo daños mayores (24).

El diagnóstico enfermero orientado a la vigilancia (DOV) se clasifica en cinco tipos:

- DoV primaria: identifica una situación que requiere vigilancia y control de factores causales o condicionantes de salud. Ejemplos:

Riesgo cardiovascular

Conductas sexuales de riesgo

- DoV secundaria: identifica una situación que requiere cribado y detección precoz. Ejemplos:

Riesgo de desatención infantil

Riesgo de síndrome ansioso depresivo

- DOV terciaria: identifica una situación que requiere seguimiento, estabilización y rehabilitación. Ejemplos:

Riesgo de descompensación/exacerbación

Riesgo de recurrencia/progresión de la infección

- Dov cuaternaria: identifica una situación que requiere prevención, identificación y actuación precoz ante posibles consecuencias de las intervenciones sanitarias (iatrogenia). Ejemplos:

Riesgo de extravasación

Dolor procedimental

- DOV crítica: identifica una situación que requiere prevención activa, identificación y actuación precoz del deterioro agudo. Ejemplos:

Riesgo de recurrencia/progresión del delirio

Riesgo de shock cardiogénico 


\section{Cuadro 2. Red conceptual sobre el diagnóstico enfermero en ATIC (cont.)}

\section{Diagnóstico orientado a la autonomía}

Juicio clínico (o la conclusión de varios juicios) sobre el estado y la capacidad del individuo (familia, grupo o comunidad) de escoger sus propias opciones según sus convicciones, razonamientos y objetivos, en el contexto de la relación e interacción con los demás, por los que también es autónomo aquel que es capaz de identificar a las personas (o elementos) de quien depende, en términos de autocuidado para las actividades de la vida diaria, desarrollo personal, voluntad y toma de decisiones (11). Ejemplos:

Riesgo de deterioro funcional

Riesgo de claudicación familiar

\section{Diagnóstico orientado al bienestar y al control de síntomas}

Juicio clínico (o la conclusión de varios juicios) sobre la necesidad de la persona (familia, grupo o comunidad) de mejorar su equilibrio integral, su nivel de confort físico, emocional, social y/o espiritual y aliviar el sufrimiento. Ejemplos:

Fatiga

Duelo familiar

\section{Diagnóstico de lesiones y dolencias físicas}

Juicio clínico (o la conclusión de varios juicios) sobre el daño a la integridad tisular localizado sin afectación sistémica o la presencia de indisposiciones, afecciones físicas o enfermedades menores que se autolimitan y tienen una afectación de la vida diaria limitada en el tiempo. Úlcera del pie diabético grado II

Resfriado común

\section{Diagnóstico de emergencia y de actuación urgente}

Juicio clínico (o la conclusión de varios juicios) sobre la presencia de un problema agudo que representa una amenaza inminente para la vida o la integridad de la persona (familia, grupo o comunidad). Ejemplos:

Paro cardiorrespiratorio Violencia

Nota 1. Estas tipologías no son autoexcluyentes. Un diagnóstico puede ser a la vez de agrupación, principal y orientado a la vigilancia; por ejemplo, en el caso de un recién nacido sano a término, el diagnóstico Inmadurez fisiológica del neonato.

Nota 2. Las referencias utilizadas en el Cuadro 2 se pueden ver en el apartado bibliografía.

Los elementos que apoyan el diagnóstico, es decir, factores de riesgo, causales, predisponentes, desencadenantes y manifestaciones, han de estar registrados en la valoración y no necesariamente explicitados como parte de la etiqueta diagnóstica (5). El PES tiene gran importancia en el aprendizaje de los ejercicios deliberativos de juicio clínico y, por ello, sigue siendo una herramienta docente de primer orden, pero esta relevancia no debería trasladarse linealmente al registro asistencial.

El uso de los diagnósticos de esta terminología fue evaluado en un estudio multicéntrico, con una muestra de 246.000 pacientes, en el que se comprobó que el 92\% de los conceptos diagnósticos incluidos en ATIC es utilizado por las enfermeras para reflejar sus juicios clínicos (16).

En términos de precisión diagnóstica, el estudio de González Samartino (23) ha demostrado que las enfermeras registran con mayor precisión que los médicos, los diagnósticos de lesiones por presión y con precisión similar el diagnóstico de infección de herida quirúrgica. Del mismo modo, en otro estudio se ha evaluado la utilidad de los diagnósticos orientados a la vigilancia, observándose que los pacientes, con este tipo de diagnósticos identificados, tenían mejores resultados en términos de detección precoz de complicaciones críticas y de resultados de supervivencia (24).

El contenido del eje diagnóstico de ATIC también ha sido evaluado en términos de cobertura conceptual para representar los diagnósticos en la gestión enfermera de la demanda en Atención Primaria. Los resultados de este análisis indican que ATIC ofrece mayor cobertura conceptual para representar los juicios que las enfermeras emiten en el cuidado a personas con dolencias agudas en Atención Primaria (34).

\section{Intervención}

El Eje Intervención de ATIC contiene actuaciones cuidadoras autónomas y de colaboración, y sus especificaciones relacionadas.

La conceptualización de las intervenciones enfermeras, apoyada en la misma fundamentación filosófica y teórica (10-12), establece que a efectos de la representación de la prestación de cuidados una intervención es la "prescripción y realización de una prestación enfermera que deriva del diagnóstico y refleja el abordaje para su prevención, resolución o paliación, total o parcial. (...) Las especificaciones permiten la representación de elementos esenciales que caracterizan la intervención" (5). En este sentido, una intervención sería lo "que se hace" y un procedimiento sería el "cómo se hace". En la práctica, el registro de la prestación de intervenciones no requiere todo el detalle del "cómo se hace"; precisa el nivel de especificidad necesario para caracterizar la intervención y su realización en cada caso concreto.

En ATIC, la definición de cada intervención explicita cuáles son los objetivos que se persiguen (Cuadro 3). Con esta integración se consigue una representación implícita de los objetivos específicos de los cuidados en los registros. Las intervenciones también pueden contener orientaciones sobre las indicaciones, contraindicaciones, posibles complicaciones y especificaciones relacionadas más habituales, aunque en la práctica asistencial, las enfermeras solo utilizan la denominación de la intervención (y de las especificaciones 
Cuadro 3. Ejemplo del contenido de una Intervención ATIC

ACTIVIDAD FÍSICA

$2017(1993)$

IE que tienen como objetivo: fomentar, capacitar o asistir mínimamente en la realización de movimiento corporal estático o con desplazamiento para aumentar el gasto energético y el trabajo muscular de la persona en beneficio de su salud.

La actividad física puede ser espontánea (caminar, realizar las actividades de la vida diaria básicas e instrumentales) o programada (practicar cualquier deporte, yoga u otras actividades sociales que implican movimiento) y contribuye a un estilo de vida saludable.

La actividad aeróbica o de resistencia (correr, nadar, ir en bicicleta) aumenta la frecuencia cardiaca y respiratoria y moviliza los grandes grupos musculares. La actividad física de fortalecimiento musculoesquelético (subir escaleras, hacer flexiones, abdominales o pesas, saltar) mejora la resistencia, la fuerza y la potencia y, a su vez, también puede ser aeróbica. Los estiramientos y el yoga mejoran la flexibilidad, la movilidad de las articulaciones y el control de la respiración.

Precoordinados: [10005471] Actividad física: estimular; [10006815] Actividad física: instruir; [1 0005577] Actividad física: supervisar.

Sinónimos: [10005576] Ejercicio físico: estimular (CS); [10005574] Educación sanitaria: actividad física (S); [10005575] Ejercicio físico: supervisar (CS).

\section{Selección de especificaciones:}

$10001771 \quad$ EAM pasivos de hemicuerpo inferior

$10002177 \quad$ Ejercicios de flexo-extensión

$10002175 \quad$ Ejercicios de contracción-relajación

$10002012 \quad$ Programación actividad-reposo Instruir

10004782 Tolerancia a la actividad Valorar

(...)

\begin{tabular}{|c|c|}
\hline $\begin{array}{l}\text { Target } \\
\text { Medio } \\
\text { Localización }\end{array}$ & $\begin{array}{l}\text { Actividad física } \\
\text { Implícito } \\
\text { NP }\end{array}$ \\
\hline $\begin{array}{l}\text { Dimensión } \\
\text { Componente } \\
\text { Categoría } \\
\text { Metaconcepto }\end{array}$ & $\begin{array}{l}\text { Cuidados integrales } \\
\text { Actividad y movimiento } \\
\text { Conductas y hábitos saludables } \\
\text { Actividad física }\end{array}$ \\
\hline $\begin{array}{l}\text { PCE } \\
\text { AID } \\
\text { Composición }\end{array}$ & $\begin{array}{l}\text { Abundante } \\
\text { Todas } \\
\text { Compuesto }\end{array}$ \\
\hline
\end{tabular}

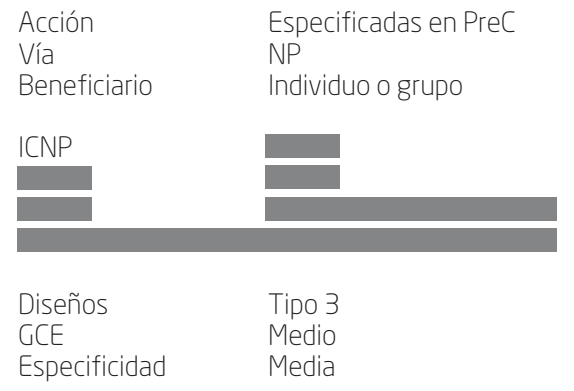

Criterios de resultados y parámetros de evolución

Actividad física realizada

Actividad física tolerada

Mejora autopercibida de la condición física

Disminución o ausencia de fatiga

Incremento o mantenimiento de la masa muscular

Mejora o mantenimiento del patrón de sueño

Instruir
Valorar

\section{Selección de referencias}

(...)

Juvé ME . ATIC Eje Intervención. Próxima publicación 2018. Reproducido con autorización de la autora

Nota. Algunas partes se han ocultado para preservar la fuente original. IE: intervención enfermera. CS: cuasi-sinónimos. EAM: ejercicios de amplitud de movimiento. PreC: precoordinados. PCE: producción científica enfermera. AID: áreas de interés disciplinar. GCE: grado de complejidad estándar de la intervención

necesarias) como elemento para informar de todo ello en los registros $(17,18)$.

La conceptualización de la práctica asistencial enfermera en ATIC determina que esta "se fundamenta principalmente en la optimización del estado de salud y de la prevención de su deterioro. En la salud, para su preservación; en los estados de salud relativa, para prevenir la enfermedad y promover el restablecimiento del estado previo; en los estados de enfermedad, para prevenir complicaciones o su progresión y promover el restablecimiento o consecución del mejor estado de salud posible; y al final de la vida, para prevenir y paliar el sufrimiento" (12). Bajo esta óptica, puesto que la principal responsabilidad de la enfermera es ayudar a la persona (familia, grupo o comunidad) a mantener o restablecer su estado de salud, el concepto de "orden médica" se asimila al de una solicitud de un profesional sanitario a otro en una relación simétrica de igual a igual (35). Por ello, las prescripciones de cuidados son indicadas por las enfermeras de forma autónoma, pero algunas intervenciones también pueden ser indicadas como resultado del pacto de cuidados entre la enfermera y el médico u otro profesional, en el marco de la corresponsabilidad del abordaje de determinados problemas de salud $(5,8)$.

De igual modo, la ejecución de una intervención puede ser llevada a cabo por el beneficiario de los cuidados o sus cuidadores de forma autónoma, o con educación, ayuda, colaboración o supervisión de la enfermera; por la enfermera de forma autónoma supliendo al paciente; por otro profesional con la colaboración de la enfermera; o bien ser delegada al personal auxiliar 
(5,8). Algunos estudios recientes aportan evidencias sobre el eje intervención de la terminología ATIC. El primero cuyo objetivo era evaluar la frecuencia de uso de intervenciones psicoemocionales en una muestra de 150.000 pacientes, demuestra que el $98,4 \%$ de las intervenciones de estas categorías de ATIC es empleada en la práctica para representar la prestación de cuidados psicoemocionales a pacientes, padres y cuidadores, siendo la Escucha activa (93,8\%) y la Estabilización emocional (82,8\%) las intervenciones más habituales (21).

La evaluación del grado de conocimientos de las intervenciones de vigilancia ATIC en una muestra de 409 enfermeras, evidenció que un 84,2\% de las participantes tenía un nivel de conocimientos intermedio o alto y que menos de un 5\% tenía un nivel de comprensión bajo $(23,36,37)$.

En otro estudio multicéntrico, que evaluó la coherencia entre el registro del diagnóstico enfermero, las intervenciones y los resultados en una muestra de 44.932 pacientes, los resultados demostraron una elevada correspondencia $(76,7 \%)(23,28)$.

\section{Consideraciones finales}

Podría argumentarse que las evidencias aportadas sobre el uso de ATIC tienen una fortaleza moderada puesto que se sustentan en diseños observacionales descriptivos. Sin embargo, la última revisión sistemática en relación al uso de los lenguajes enfermeros indica que entre el 60\% y el 87,7\% de estudios sobre las clasificaciones enfermeras tradicionales son observacionales (38).

La terminología ATIC empezó a utilizarse en la práctica asistencial en 2007, por lo que en este sentido tiene un recorrido más breve las clasificaciones. Es lógico pensar que convendría realizar más estudios analíticos comparando los resultados de uso de los distintos lenguajes.

Las consideraciones expuestas sobre el empleo de ATIC en la práctica han de interpretarse en relación a la fundamentación que sustenta el desarrollo de este vocabulario controlado, no solo desde las evidencias generadas mediante su uso. Esta aportación conceptual no pretende establecer dogmatismos, ni resta valor a la labor erudita de muchas enfermeras académicas y asistenciales que han participado del desarrollo de otros vocabularios controlados.

ATIC intenta contribuir a facilitar y optimizar los registros de la prestación de cuidados que realizan las enfermeras en la práctica asistencial, para hacer más visible la aportación y el impacto de los cuidados enfermeros a los resultados de salud de las personas.

\section{Financiación \\ Ninguna.}

\section{Conflicto de intereses}

La autora de este artículo es a su vez autora de la terminología ATIC.
Desde 1992, el desarrollo de esta terminología se ha sustentado con la aportación individual de su autora y no ha recibido financiación alguna por parte de ningún organismo ni institución.

\section{$\overline{\text { Bibliografía }}$}

1. Pijoan B. La enfermera moderna. $2^{a}$ ed. Barcelona: Lib. Sintes; 1938.

2. Institutes of Medicine (IOM). The Future of Nursing. Leading change, Advancing Health. Institute of Medicine (US). Washington DC: The National Academies Press; 2011.

3. Heartman TH. NANDA Internacional Diagnósticos enfermeros: definiciones y clasificación 2015-2017. Barcelona: Elsevier; 2015.

4. Juvé Udina ME. La terminología ATIC. Barcelona: Naaxpot SLU; 2016.

5. Juvé Udina ME. Evaluación inductiva de la estructura de una terminología de interfase: conceptualización del proceso enfermero. Nursing (ed. española). 2012; 30(7):62-6.

6. Thorne S, Canam C, Dahinten S, Hall W, Henderson A, Reimer-Kirkham S. Nursing's metaparadigm concepts: disimpacting the debates. J Adv Nurs.1998; 27(6): 1257-68.

7. Brito Brito PR. Redes y diagnósticos enfermeros. Almería: Ed. Círculo Rojo; 2015.

8. Juvé Udina ME. Evaluación de la validez de una terminología de interfase. Tesis doctoral. Universidad de Barcelona, 2012.

9. Juvé Udina ME. La terminología ATIC [internet]. [citado 3 nov 2017]. Disponible en: http://www.atic.com.es/

10. Juvé Udina ME. Terminología enfermera de interfase. Fundamentos filosóficos y teóricos para su desarrollo y validación. Rev ROL Enferm. 2012; 35(4):260-5.

11. Juvé Udina ME. ATIC. Una terminología de interfase. Rev ROL Enferm. 2012; 35(5):368-75.

12. Juvé Udina ME. Salud, entorno y Enfermería. Fundamentos filosóficos y teóricos para el desarrollo y la validación de una terminología enfermera de interfase. Rev ROL Enf. 2012; 35(6): 409-16.

13. Juvé Udina ME. A nursing interface terminology. EvaIuation of face validity. Open J Nurs. 2012; 2(3):196203.

14. Juvé Udina ME. Is the ATIC terminology oriented to nursing phenomena? Open J Nurs. 2012; 2(4):388-95.

15. Juvé Udina ME, González Samartino M, Matud Calvo C. Mapping the diagnosis axis of an interface terminology to the NANDA International Taxonomy. ISRN Nurs. 2012; ID676905.

16. Juvé Udina ME. What patients' problems do nurses e-chart? Longitudinal study to evaluate the usability of an interface terminology. Int J Nurs Stud. 2013; 50:1698-710.

17. Juvé Udina ME (ed.). Plans de cures estandarditzats per malalts hospitalitzats [internet]. Generalitat de Catalunya, Departament de Salut, Institut Català de la Salut 
2013. [citado 3 nov 2017]. Disponible en: http://ics. gencat.cat/web/.content/documents/Planscures.pdf

18. Castellà Creus M, Creus Macià MJ, Díez Sánchez B, Martí Carrasco N, Barberà Llorca MA, Andrés Martínez I. Proceso de individualización del plan de cuidados estandarizado en un sistema de información. Caso clínico: Ictus. Nursing (ed. esp.). 201 1; 29(9):60-6.

19. Romero García M, Juvé Udina ME, Fabrellas Padrés N, González Samartino M, Delgado Hito P, de la Cueva Ariza L. Glosario de términos de metodología y lenguajes enfermeros. Documento académico. [internet]. Universidad de Barcelona, 2015. [citado 3 nov 2017]. Disponible en: http://diposit.ub.edu/dspace/handle/2445/ 63294?mode=full

20. Kalenderian E, Walji M, Ramoni R. Applying HCl principles is designing usable systems for dentistry. In: Patel VL, Kannampallil TG, Kaufman DR (eds.). Cognitive Informatics for Biomedicine. Cham (Suiza). Springer International Pub. 2015. p. 189-213.

21. Juvé Udina ME, Zuriguel Pérez E, Fabrellas Padrés N, González Samartino M, Romero García M, Castellà Creus M, et al. Basic nursing care: retrospective evaluation of communication and psychosocial interventions documented by nurses in the acute care setting. J Nurs Scholarsh. 2014; 46(1):65-72.

22. Solà Miravete E. Valoración enfermera y factores de riesgo en la prevención del delirio en la persona de edad avanzada hospitalizada. Tesis doctoral. Universitat Rovira i Virgili; 2017.

23. González Samartino M. Influencia del uso de la terminología ATIC en la seguridad de los enfermos ingresados: impacto en la comunicación profesional y los resultados clínicos. Tesis doctoral. Universitat de Barcelona; 2017.

24. Juvé Udina ME, Fabrellas Padrés N, Adamuz Tomás J, Cadenas González S, González Samartino M, de la Cueva Ariza L, et al. Surveillance nursing diagnoses, ongoing assessment and outcomes on in-patients who suffered a cardiorespiratory arrest. Rev Esc Enfermagem USP (En prensa. Aceptado para publicación septiembre 2017)

25. Juvé Udina ME, Fabrellas Padrés N, Delgado Hito $P$, Hurtado Pardos B, Martí Cavallé M, Gironés Nogué M, et al. Newborn physiological immaturity: A concept analysis. Adv Neonatal Care. 2015; 15(2):86-93.

26. Juvé Udina ME, Matud Calvo C, Vila Batllori N. Model de dades de la prestació de cures a malalts hospitalitzats. Guía de consulta. Institut Català de la Salut [internet], 2015. [citado 3 nov 2017]. En: http://ics.gencat.cat/ web/.content/documents/assistencia/informes_dui.pdf

27. Agustino S, Planas MA, Tapia M, Berbis C, Muñoz A, Juvé-Udina ME. From admission to discharge. What assessment data do nurses chart using the ATIC Terminology. Barcelona: ICN Congress Proceedings P234S; 2017.

28. Juvé Udina ME. Desarrollo de un sistema de valoración clínica basado en la teoría de la complejidad y la ciencia enfermera. Nursing (ed. española) 2005; 23(5):50-5.
29. Solà Miravete E, López C, Martínez Segura E, Adell Lleixà M, Juvé Udina ME, Lleixà Fortuño M. Nursing assessment as an effective tool for the identification of delirium risk in older in-patients: a case-control study. J Clin Nurs 2017, DOl: 10.1111/jocn.13921.

30. Adamuz Tomás J, López Jiménez MM, Tapia Pérez M, González Samartino M, Matud Calvo C, Juvé Udina ME. ¿Identificamos la complejidad de los pacientes hospitalizados en la valoración enfermera? Libro de ponencias 16428. A Coruña: XX Encuentro Internacional de Investigación en Cuidados; 2016.

31. Juvé Udina ME, Matud Calvo C, Farrero Muñoz S, Jiménez Pérez H, Rodríguez Guías E, Martínez Muñoz M, et al. Intensidad de cuidados ¿cargas de trabajo o complejidad individual? Metas Enferm. 2010; 13(8):6-14.

32. Adamuz Tomás J, López Jiménez MM, Jiménez Martínez E, Pérez Tapia M, González Samartino M, Juvé Udina ME. Patient complexity and risk factors associated with readmissions. Barcelona: ICN Congress Proceedings C081S; 2017.

33. Lake S, Moss C, Duke J. Nursing prioritization of the patient need for care: a tacit knowledge embedded in the clinical decision-making literature. Int J Nurs Pract. 2009; 15:376-88.

34. Ríos AM, Fabrellas N, Amat G, Artigas M, Juvé Udina ME. Nurse-led same day visit for acute minor illnesses: Comparing concept coverage of ICD-10, NANDA and ATIC to represent nursing diagnoses in Primary Care. Barcelona: ICN Congress Proceedings P066T; mayo 2017.

35. Pijl Zieber EM. Doctors'order and the language representation. Nurs Philosophy. 2013; 14(2):139-47.

36. González Samartino M, Delgado Hito P, Adamuz Tomás J, Matad Calvo C, Tapia Pérez M, López Jiménez MM, Juvé Udina ME. Una terminología interfase como eje de unos cuidados seguros. Nivel de conocimientos y comprensión de intervenciones enfermeras ATIC. Rev Rol Enf. 2017; 40(10):698-709.

37. González Samartino M, Delgado Hito P, Juvé Udina ME. Precisión y exhaustividad del registro de eventos adversos mediante una terminología de interfase. Rev Esc Enfermagem USP (En prensa) [Aceptado para publicación octubre, 2017].

38. Tastan S, Linch GCF, Keenan GM, Stiffner J, McKinney $D$, Fahey $L$, et al. Evidence for the existing American Nurses Association-recognized standardized nursing terminologies: A systematic review. Int I Nurs Stud. 2014; 51:1160-70.

Si quieres ampliar más información sobre este artículo, consulta:

\begin{tabular}{|c|c|}
\hline $\begin{array}{l}\text { Introduce estos } \\
\text { términos: }\end{array}$ & $\begin{array}{l}\text { Valoración enfermera; administración de } \\
\text { servicios de salud; historia clínica electróni- }\end{array}$ \\
\hline encuentr@ & ca; documentación clínica. \\
\hline
\end{tabular}

Contacta con los autores en:

lalajuve.on@gmail.com 